\title{
Quarterly intrinsic disorder digest (January-February-March, 2014)
}

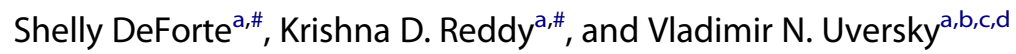

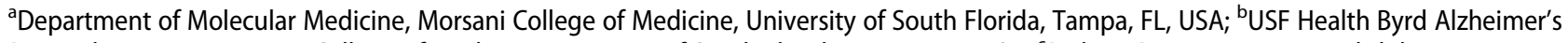 \\ Research Institute, Morsani College of Medicine, University of South Florida, Tampa, FL, USA; 'Biology Department, King Abdulaziz University, \\ Jeddah, Kingdom of Saudi Arabia; 'Laboratory of Structural Dynamics, Stability and Folding of Proteins, Institute of Cytology, Russian Academy \\ of Sciences, St. Petersburg, Russia
}

\section{ABSTRACT}

This is the $5^{\text {th }}$ issue of the Digested Disorder series that represents a reader's digest of the scientific literature on intrinsically disordered proteins. We continue to use only 2 criteria for inclusion of a paper to this digest: The publication date (a paper should be published within the covered time frame) and the topic (a paper should be dedicated to any aspect of protein intrinsic disorder). The current digest issue covers papers published during the first quarter of 2014; i.e., during the period of January, February, and March of 2014. Similar to previous issues, the papers are grouped hierarchically by topics they cover, and for each of the included papers a short description is given on its major findings.

\section{KEYWORDS}

database; intrinsically disordered proteins; protein function; structural ensemble; structural proteins

\section{Introduction}

This article continues the Disorder Digest series. ${ }^{1-4}$ The goal of this series is to provide an unbiased and condensed survey of the literature on intrinsically disordered proteins on a quarterly basis. As in the previous issues, no special filtering was used except to verify the print date, and exclude those papers not related to the topic. The digest article is structured hierarchically and papers are grouped in several sections: (1) structures of intrinsically disordered proteins (IDPs); (2) functions of IDPs; (3) methods for the IDP analysis; (4) proteomics of IDPs; (5) IDPs and diseases; and (6) IDPs/IDPRs as drugs or drug targets. One should keep in mind that the unambiguous classification of many papers is challenged by the intertwining of the topics they cover.

In the $5^{\text {th }}$ digest of this series, we cover papers published in January, February, and March of 2014. We used the following search term in PubMed: (intrinsically OR natively OR naturally OR inherently) AND (disordered OR unfolded OR unstructured OR denatured) AND (protein OR region OR peptide OR domain) AND (“2014/01”[PPDAT]: “2014/ 03”[PPDAT]), which returned 103 hits. After filtering hits not relevant to the topic, we have covered 94 articles here. Figure 1 represents a word cloud, which shows the most represented words from all the abstracts of papers included in this issue.

We also introduce below a new rubric "Spotlight" that specifically emphasize some of the most interesting and important developments in the field of intrinsically disordered proteins during the covered quarter.

\section{Spotlight: IDP databases and the rise of the ensemble view}

Databases of experimentally verified IDPs are critical for the development of tools that predict intrinsic disorder, and provide an important data set for the study of IDP functions, motifs and structural behavior. One of the first databases to provide experimental evidence of intrinsic disorder was oddly enough, the Protein Data Bank, where regions of missing electron density in $\mathrm{x}$-ray crystal structures provide an indication of possible disorder. The major databases for IDPs are Disprot $^{5}$ and the recently updated IDEAL ${ }^{6}$ and the mostly predictor based databases $\mathrm{d} 2 \mathrm{p} 2^{7}$ and MobiDB. ${ }^{8}$

CONTACT Vladimir N. Uversky $\otimes$ vuversky@health.usf.edu $\Theta$ University of South Florida, Department of Molecular Medicine, 12901 Bruce B. Downs Blvd., MDC07, Tampa, FL 33612, USA.

\# These authors equally contributed to this work.

(c) 2016 Taylor \& Francis 


\section{acid activity aggregation binding biology conformational disordered domain dynamics effects

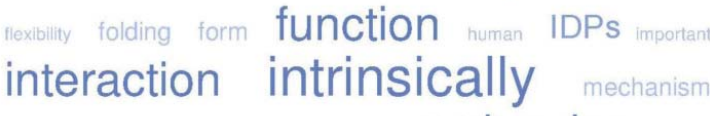 medicine membrane methods model molecular OrOteingregions regulation residues ma role sciences sequence Structural}

Figure 1. A Wordle for the most abundant words in the abstracts of IDP-related papers published during the first quarter of 20014. The size of each word is increased in proportion to its number of occurrences.

pE-DB provides an entirely new kind of database of IDPs, providing structural ensembles of IDPs. ${ }^{9}$ Tompa and Varadi discuss the predictive power of these ensembles, ${ }^{10}$ pointing to the challenges inherent in interpreting and choosing the correct ensembles. However, new methods to provide atomic resolution descriptions of IDPs, such as those outlined by Schwalbe et al. ${ }^{11}$ as well as new tools for visualizing conformational variation ${ }^{12}$ indicate that we may soon see an explosion of ensemble-based analysis.

\section{Studies on structural properties of IDPs and IDPRs}

The proliferating-cell-nuclear-antigen-associated factor $\mathrm{p} 15^{\mathrm{PAF}}$ is a nuclear protein highly expressed in several types of cancer. p15 $5^{\mathrm{PAF}}$ is likely associated with cancer by binding the transactivation region of $\mathrm{p} 53$ and therefore inhibiting transcriptional activity. De Biasio et al. structurally characterized this protein using several structural techniques including NMR, CD spectroscopy, and SAXS, and ultimately found that $\mathrm{p} 15^{\mathrm{PAF}}$ is an IDP with several regions of transient secondary structure which coincide with sites of posttranslational modification and protein-protein interactions. ${ }^{13}$ This suggests a mechanism where intrinsic disorder plays a role in $\mathrm{p} 15^{\mathrm{PAF}}$-associated cancer.

Skp1 is an IDP that serves as an adapter in the Skp1/cullin1/F-box protein family of E3 ligases, and is hydroxylated and subsequently glycosylated. Using several biochemical and biophysical strategies, Sheikh et al. investigated the role of glycosylation on Skp1, and found that this modification alters the secondary structure content of the protein, thereby influencing Skp1-F-box protein interactions. ${ }^{14}$

The solute carrier gene family (SLC26) have multiple cellular functions which all involve halide. Keller et al. analyzed this family and found that SLC26 proteins have intrinsically disordered C-termini which are predicted to bind calmodulin $(\mathrm{CaM}) .{ }^{15}$ Using prestin (SLC26A5), a voltage-operated motor and weak transporter, as a model, the authors found that its Cterminus binds CaM. This calcium-dependent interaction hyperpolarizes the operating point of the protein, which results in differential states of individual prestin molecules. The authors use bioinformatics to infer that the members of the SLC26 family have the same mechanism.

Several IDPs undergo a folding-upon-binding transition, where transient secondary structure is formed prior to the bound, ordered state. To determine the effect of this transient structure on binding kinetics, Iesmantavicius et al. made a number of mutations in the activation domain of ACTR (activator of thyroid hormone and retinoid receptors) which did not affect interaction residues but did affect helicity. ${ }^{16}$ The authors found that indeed, the transient secondary structure prior to bound secondary structure is important for disorder-to-order transitions of IDPs.

$\alpha$-synuclein is a well-established IDP that interacts with membranes. Lee et al. explored the dependence of $\alpha$-synuclein structural transitions on membrane lipid content using several biophysical techniques. ${ }^{17}$ They provide an in-depth analysis of the correlation between polarity content of the membrane and electrostatic vs. hydrophobic interactions, which may increase understanding of $\alpha$-synuclein interactions and aggregation in the presence of membranes.

Like $\alpha$-synuclein, $\gamma$-synuclein is thought to be intrinsically disordered. Golebiewska et al. determined the native state of the $\gamma$ isoform using a combination of biochemical and biophysical techniques. ${ }^{18}$ Recombinant $\gamma$-synuclein was found to be monomeric; however, when its physiological binding partner PLC $\beta 2$ or membranes are introduced, $\gamma$-synuclein dissociates to monomeric form. This result was also reconstituted in cells using microinjection. Therefore, it is proposed that $\gamma$-Synuclein oligomer-to-monomer transitions (and vice versa) may serve to modulate function. 
Tau is an IDP associated with Alzheimer's disease, and the numerous serines and threonines found on tau are competitively post-translationally modified by phosphorylation and OGlcNAcylation. Brister et al. used a number of biophysical techniques on peptides derived from the proline-rich domain of tau, and found that while phosphorylation promoted polyproline helix formation, OGlcNAcylation had the opposite effect. ${ }^{19}$ Additionally, the authors found that pThr had greater effects on polyproline helix promotion than pSer, likely because of both higher disorder of nonphosphorylated Thr and more order for pThr.

Schwalbe et al. used a combination of SAXS, NMR, and the genetic algorithm ASTEROIDS to generate an atomic-resolution ensemble of tau and $\alpha$-synuclein. ${ }^{11}$ The ensemble was confirmed using cross-validation from independent experimental datasets. Besides the description of local and long-range conformational information, the authors found that polyproline II is preferred in regions of nucleation, potentially providing a structural basis of aggregation propensity.

Grishin et al. used a combination of biochemical assays and X-ray crystallography to elucidate the first structure of family of bacterial effector kinases, $\mathrm{NIeH} .{ }^{20}$ They found that the N-terminal segment is intrinsically disordered, and is autophosphorylated by the C-terminal kinase domain. Additionally, while these proteins retain kinase activity, the authors found several significant differences between the NIeH family and classical kinases, including a shortened activation loop and a missing C-terminal segment.

The HIV-1 gag polyprotein is essential for viral assembly; however, only structures of the individual components were previously obtained because of conformational heterogeneity and poor crystallization properties of the longer chains. Using solution NMR spectroscopy, Deshmukh et al. described a 300 residue capsid-SP1-nucleocapsid (CA-SP1-NC) fragment, and find that the individual subunits behave similarly to previously described isolated structures. ${ }^{21}$ Additionally, the authors found that nucleic acids exclusively bind to the NC domain, while the C-termini of CA and SP1 are both intrinsically disordered.

The retinoic acid nuclear receptor (RAR) is difficult to recombinantly purify, partly because of a biologically significant $\mathrm{N}$-terminal domain containing a proline-rich domain that is predicted to be intrinsically disordered. Martinez-Zapien et al. described a purification protocol which combines a maltose binding protein solubility tag and strategic centrifugation to produce a soluble RAR fragment, which they verified with several biophysical techniques. ${ }^{22}$

The concept of 'fuzziness', where IDPs participate in multivalent interactions without undergoing a disorder-to-order transition, is a widely debated idea. A study by Nourse and Mittag addressed the T-cell receptor zeta subunit, which had been previously thought to form disordered dimers. ${ }^{23}$ Using several biochemical and biophysical techniques, the authors found that instead, this protein is monomeric in solution unless driven to extremely high concentrations. Instead, previous observations interpreted to be 'fuzziness' were instead likely to be caused by abnormal interactions with chromatography resins, which raises the question if 'fuzziness' exists in nature.

Spectrin, a structural protein required for maintenance of the erythrocyte cytoskeleton, are composed of heterodimers consisting of $\alpha$ and $\beta$ subunits. The C-terminus of the $\beta$-subunit interacts with the $\alpha$-subunit non-covalently, which is called the tetramerization domain. Hill et al. found that this tetramerization domain is highly stable, and used protein engineering to describe the assembly mechanism of this domain. ${ }^{24}$ The authors ultimately found that this mechanism is distinct from other known folding mechanisms of spectrin domains.

Butz et al. used a combination of yeast surface display and fluorescence-activated cell sorting to optimize Spider Roll, a computationally engineered protein designed to recognize the p21-activated kinase 1 (PAK1). ${ }^{25}$ The authors obtained variants which displayed tighter binding, but surprisingly, the representative variant (MSR7) was partially disordered. This finding has interesting implications for computational design of proteins, which do not generally consider intrinsic disorder, yet an experimentally optimized protein displayed intrinsic disorder.

Deleted in breast cancer 1 (DBC1/CCAR2) regulates a number of cellular processes through multivalent interactions. Cell cycle and apoptosis regulator 1 (CCAR1) is a paralog with similar functions, domains, and can cooperatively bind to DBC1. Brunquell et al. used various computational techniques to predict structure and correlate to evolutionary properties, and found that $\mathrm{DBC} 1$ and CCAR2 are both intrinsically disordered in a conserved fashion. Additionally, the authors found that $C$. elegans LST-3 is likely to be the ancestral sequence of these genes. 
Microtubule-associated proteins (MAPs) control the functions of microtubules by controlling their dynamics. Hashi et al. created a minimal active fragment of MAP4 in order to make this protein sizecompatible with NMR, and found that MAP4 is an IDP that binds to a microtubule in a multivalent manner, using its multiple binding sites. ${ }^{26}$ This observation contradicts previous data, which indicated that multiple binding sites acted simultaneously on multiple microtubules.

The cellular environment is crowded by a number of macromolecules, which can influence local protein concentrations and conformations. While efforts have been made to mimic this environment experimentally, the polymers used are generally neutral and flexible, and do not fully capture the diversity of the cellular environment. Breydo et al. examined the effect of more rigid polymers on several proteins encompassing a range of folded states. ${ }^{27}$ The authors found that these polymers affect protein aggregation and folding via multiple mechanisms, including not only excluded volume effect, but also local viscosity and protein-polymer interactions.

The folding of retro-domain proteins, or proteins with reversed sequence, are of considerable debate in the protein folding field. Kutyshenko et al. examined the normal and retro forms of SHH, a Src homology 3 (SH3) domain-containing protein whose normal sequence is highly stable and requires no extraneous factors such as disulfide bonds for proper folding. ${ }^{28}$ The authors found that retro-SHH does not contain stable globular structure, and instead has residual secondary structure. The structures of normal and retro-SHH react differently to dodecylphosphocholine micelles, and predictions indicated that reversal of sequence did not affect propensity toward intrinsic disorder.

The mammalian SLC9A family of $\mathrm{Na}^{+} / \mathrm{H}^{+}$exchangers control $\mathrm{pH}$ within organelles, cytoplasm, and the extracellular environment. While a wealth of biochemical and structural information exist for this family, there is still no crystal structures on any SLC9A protein. Hendus-Altenburger et al. reviewed the current state of the field in terms of structure and regulation. ${ }^{29}$ Additionally, the authors used bioinformatical and structural analyses to evaluate models of SLC9A ion transport.

\section{Analyzing functions of IDPs and IDPRs}

The many biological functions that are aided by intrinsic disorder are outlined in a comprehensive review published this quarter by Oldfield and Dunker which also covers the history of the field, common experimental and computational methods, implications in evolution, and future directions. ${ }^{30}$ While the relationship between biological function and intrinsic disorder is well established, some of commonly stated advantages of intrinsic disorder, such as high specificity, low affinity binding, have been difficult to demonstrate experimentally until recently. Hisaoka et al. used chemical cross-linking experiments and fluorescent labeling to show that an intrinsically disordered and highly basic region on the nucleolar protein B23 binds RNA with high affinity and low specificity. Furthermore, this activity appears to be regulated by interactions with an adjacent acidic region. ${ }^{31}$ Intrinsic disorder is also considered an advantage in promiscuous binding, due to its adaptability, however experimental quantifications of this advantage have been rare. Using a number of differently stable variants, Quan et al. $^{32}$ were able to establish a relationship between an increase in instability in the Spy chaperone, and the improvement in chaperone activity. Kalinowska et al. discuss intrinsically disordered proteins in the context of the "fuzzy oil drop" model which describes a set of properties over an entire molecule that lead to a hydrophobic core surrounded by a hydrophilic mantle that interacts with water and stabilizes the core. They show that the water environment influences the positions of disordered fragments, and examine DisProt ${ }^{5}$ to show examples that both fit and converge from the model.

P27Kip1 is an intrinsically disordered protein that modulates G1 progression through its interactions with cyclin-dependent kinases. Bencivenga et al. ${ }^{33}$ studied the major phosphoisoform of p27Kip1 in vivo and found that it is more stable than its unmodified form, and does not bind CDK1, possibly facilitating CDK1 activation. They propose 2 different functional paths for p27Kip1, each marked by phosphorylation on a different residue. Khurana et al. performed a series of truncation experiments on the intrinsically disordered C-terminus of Bacillus Lipase. ${ }^{34}$ Deletion of 10 residues reduced specific enzyme activity and deletion of 20 residues nearly abolished it. The authors propose that the disordered region may provide stabilization through 6 critical hydrogen bonds. Protein phosphatase 1 (PP1) controls more than $50 \%$ of all dephosphorylation reactions. Choy et al. ${ }^{35}$ examined the interaction of PP1 with one of its binding partners, 
PPQ nuclear targeting subunit (PNUTS), an IDP that tightly binds PP1 in an extended manner. They found that PNUTS blocks a substrate binding groove on PP1, and the investigation of this site allowed them to define unique PP1 binding motifs.

Bacterial-type phosphoenolpyruvate carboxylase (BTPC) is an enzyme phosphorylated at 3 sites during castor oil seed development. Hill et al. ${ }^{36}$ characterized the kinase responsible for this phosphorylation and noted that 2 of the phosphorylation sites occur in intrinsically disordered regions of BTPC, suggesting a possible for intrinsic disorder in the regulation of this enzyme.

Two papers came out this quarter on prostate-associated gene 4 (PAGE4), representing a collaboration between Johns Hopkins and North Carolina State University. PAGE4 is an intrinsically disordered stress-response protein that is aberrantly expressed in cancer. In the first study, Rajagopalan et al. ${ }^{37}$ demonstrate that c-Jun and PAGE4 interact and single-molecule FRET reveals that PAGE4 undergoes conformational changes upon binding. In the second published study, Mooney et al. ${ }^{38}$ identified the homeodomain-interacting protein kinase 1 (HIPK1) as a kinase that phosphorylates PAGE4. Single-molecule FRET shows that PAGE4 undergoes a small amount of compaction upon phosphorylation, and this conformational change results in a reduced affinity for c-Jun.

Qi et al. explored the impact of the phosphorylation of the intrinsically disordered protein osteopontin on binding to $\mathrm{UO} 2(2+)$. Their results show structural stabilization upon binding and suggest a role for osteopontin as a metal transporter in addition to its known role in bone homeostasis. Myelin Basic Protein (MBP) is an intrinsically disordered protein that is ubiquitous in the CNS, and plays an important role in the myelination of nerves by providing intermembrane adhesion between cytoplasmic leaflets. Lee et $\mathrm{al}^{39}$ used atomic force microscopy to study the adsorption of MBP to model myelin lipid bilayer membranes and found that it preferentially adheres to disordered submicron domains. Ramos et al. ${ }^{40}$ characterized the interactions of the histone chaperone Nucleoplasmin (NP) upon interaction with H3-H4 and histone octamers. Remarkably, they found through electron microscopy analysis, that the intrinsically disordered distal face of the chaperone is involved in recognition of different oligomerization states in histones.
The many functions and structural peculiarities of the glycoprotein lactoferrin are reviewed by Albar et al. Lactoferrin possesses a mix of both ordered and intrinsically disordered domains that facilitate a wide variety of antiviral and antimicrobial activities. ${ }^{41}$ The journal Molecular Endocrinology presented a review on nuclear receptors $(\mathrm{NR})^{42}$ that spotlights intrinsically disordered regions of transcription factors, including the N-terminal domain AF1. Simons et al. argue that structural flexibility is critical in the allosteric modulation of NR activity and discuss the relevance of this to therapeutic interventions. ${ }^{42}$

The nuclear pore complex mediates transport between the nucleus and the cytoplasm. It is composed of nucleoporin proteins that consist of intrinsically disordered repeating regions such as FG (PheGly) or GLFG (Gly-Leu-Phe-Gly), punctuated by more complex regions. Fiserova et al. ${ }^{43}$ used highpressure freezing to observe GLFG domains in vivo in yeast. They found both disordered and more structured states among the observed nucleoprins and proposed that dynamic movement between states may aid the movement of cargo by nucleoporins with GLFG repeats. S12 is a universally conserved protein in the small subunit of the E.coli ribosome with a non-globular region called an extension. Calidas et al. ${ }^{44}$ performed a series of truncation experiments on this extension region and found a number of deleterious effects. While the precise structure and function of this region is still not clear, this study demonstrates a necessary role for the extension of S12.

Asano et al. ${ }^{45}$ characterized the interaction between elongation factor-like 1 (EFL1) and Shwachman-Bodian-Diamond syndrome (SBDS) protein, a complex that removes Tif6 and plays a critical step in ribosome maturation. They used size exclusion chromatography, gel shift assay, and isothermal titration calorimetry and showed that the interaction is governed by the intrinsically disordered insertion domain of EFL1.

The proteasome is a multi-protein complex that is responsible for degradation of proteins. Proteins are typically marked for degradation by ubiquitin, however there are also well-characterized cases of ubiquitin independent degradation by the proteasome. Erales et al. ${ }^{46}$ reviews 3 of these cases: Rpn4, thymidylate synthase and ornithine decarboxylase. Each of these proteins has a critical intrinsically disordered region that is necessary for ubiquitin independent degradation. The authors speculate that this may be a 
historic remnant or may provide redundancy in cases of mis-regulation of the ubiquitin pool. Tomko et al. ${ }^{47}$ characterized the role of the intrinsically disordered protein yeast protein Sem1 in the formation of the proteasome. They engineered TEV protease-cleavage sites into Sem 1 and showed that Sem1 performs a tethering function in the early formation of the $26 \mathrm{~S}$ proteasome by binding Rpn3 and Rpn7, but then becomes dispensable once the ternary complex incorporates into the proteasome lid.

Certain bacteria use a similar post-translational modification of ubiquitin-like protein (Pup) to tag a protein for degradation. However, Pup, unlike ubiquitin, is intrinsically disordered. In an extensive review, Striebel et al. ${ }^{48}$ covers the current literature on the bacterial proteasome and the unique intrinsically disordered post-translational modification that signals it.

The splicesome is a large ribonucleoprotein complex that catalyzes the removal of introns from premRNA and the ligation of exons to produce mature mRNA. Christian et al. ${ }^{49}$ investigated the activation of the DEAH-box protein Prp43 by Ntr1, an interaction which promotes the helicase activity of Prp43 in the splicesome. They showed that the G-patch motif of Ntr1 is intrinsically disordered, but adopts some secondary structure upon binding to Prp43. They defined the interaction sites between Prp43 and Ntr1 using protein-protein cross-linking, protein-RNA crosslinking and hydroxyl-radical footprinting.

The kinase-inducible domain interacting (KIX) domain is a conserved 3 helix bundle that interacts with multiple transcription factors and often targets intrinsically disordered regions within transactivation domains that then undergo a disorder-order transition upon binding to a KIX region. Thakur et al. ${ }^{50}$ review the current literature on the KIX domain and its interactions. They note that several gaps remain in terms of understanding how KIX recognizes and assists in the folding of IDPs.

WH2 domains are short intrinsically disordered peptides that fold upon binding to actin. Cordon-Bleu (Cobl) regulates actin dynamics in neural development through the use of 3 adjacent WH2 domains. Jiao et al. ${ }^{51}$ used mutagenesis and electron microscopy to demonstrate that these $\mathrm{WH} 2$ repeats provide an important electrostatic environment that aids in the nucleating activities of Cobl, and that these regions are critical for filament severing activity.
The intrinsically disordered Y-box binding protein 1 (YB-1) belongs to a family of DNA- and RNA-binding proteins that contain a conserved cold shock domain. In a review by Lyabin et al. ${ }^{52}$ the diverse functions of YB-1 are discussed, including involvement in cell proliferation, stress response, and it's role in malignant cell transformation.

The binding interface of IDPs is not well characterized biophysically. In order to address this, Jemth et al. $^{53}$ used site-directed mutagenesis and binding kinetics to map the energetics of the binding interface between ACTR and NCBD. They found the interface to be highly frustrated, and postulated that promiscuous proteins cannot minimize the frustration in the binding interface to the same degree as proteins that participate in more specific interactions.

Marsh and Teichmann ${ }^{54}$ discuss the relationship between disorder and evolution at the residue level in a review. The discuss the current research and specific examples that show that local disorder is closely tied to evolutionary rate, and that there is a relationship between conformational fluctuations of an individual protein and structural variance within that protein's family.

Pre-structured motifs (PreSMos) are characterized by transient secondary structure that serves as a target-binding motif in IDPs. Interestingly, proline is often found in the flanking regions of PreSMOS. Using MD simulations, Lee et al. ${ }^{55}$ measured the influence of flanking prolines and observed a stabilizing force that suggests that prolines are encoded in PreSMos and play a stabilizing role in IDP binding.

\section{Methods for the IDP/IDPR analysis}

\section{Computational methods}

\section{Databases}

Homorepeats are common in disordered regions. Lobanov et al. followed up on their 2012 study of disordered sequence patterns and homorepeats ${ }^{56}$ and introduced a database of homorepeats for 122 proteomes that allows searching by proteome, Gene Ontology (GO) term or motif. ${ }^{57}$ Varadi et al. ${ }^{9}$ introduced pE-DB, a database of structural ensembles of IDPs. Each entry consists of primary experimental data, and the structural ensembles consistent with this data. Fukuchi et al. ${ }^{6}$ provided an update on IDEAL which has seen an expansion of its entries of IDPs from 120 to 340 . Finally, Pfam, the protein families 
database, dedicates a section in their most recent update to the challenge presented by IDPs. ${ }^{58}$ Because these regions are less well conserved, they are not easily covered by Pfam, and present a major challenge for increased coverage by Pfam.

\section{Predictors of intrinsic disorder}

The Kurgan Lab introduced 2 very different predictors this quarter. The first, Super-fast predictor of proteins with Long Intrinsically DisordERed regions, or SLIDER, is a binary predictor of proteins with long disordered regions greater than 30 residues in length. $^{59}$ The SLIDER server accepts up to 75,000 sequences at a time and returns results in a matter of hours. The second predictor is disorder based on Consensus of Predictors, or disCop, ${ }^{60}$ a consensus based predictor. Fan and Kurgan outline a method for determining the best consensus from various combinations of 20 predictors, and then implemented a binomial deviance loss-based regression to determine the consensus of a combination of 7 high performing predictors. In their tests, disCop performed significantly better than any individual predictor. Another consensus predictor was introduced this quarter, called DisMeta. DisMeta is focused on optimizing samples in preparation for NMR and X-ray crystallography and includes prediction for transmembrane domains, secretion signals and low complexity regions. ${ }^{61}$ The biannual competition for the prediction of disordered regions, called CASP10, published the results of their most recent competition this quarter. ${ }^{62}$ Among the top performers were Prdos-CNF and DISOPRED3. Overall the performance of predictors was slightly better than in CASP9.

\section{Molecular dynamics simulations}

Higo and Umeza ${ }^{63}$ present a multicanonical molecular dynamics (McMD) simulation of 2 IDP complexes: NRSF-mSin3 and pKID-KIX. Their study suggests that both induced folding and conformational selection play a role in coupled binding and folding. Another study examining the mechanism behind functionally important conformational changes was undertaken by Wang et al. ${ }^{64}$ They used a combination of normal-mode analysis and umbrella sampling to study transitions in adenylate kinase, calmodulin and p38alpha kinase upon binding to their ligands. They did not find a unified change in free energy upon ligand binding in these different systems, suggesting the conformational changes undergone by intrinsically disordered regions have remarkable complexity that is intricately related to their specific function. Rawat and Biswas $^{65}$ examined hydrogen bonds in completely disordered proteins and proteins with structured domains connected by flexible linkers. They found, unsurprisingly, that hydrogen bonds in completely disordered proteins had a shorter lifetime and show that the hydrophilic residues that are a defining feature of IDPs provide a stabilizing force maintaining dynamic equilibrium. Gerben et al. ${ }^{66}$ did a comparative study of atomistic force fields to determine which would work best with the IDP Abeta. They found that OPLS-AA, GROMOS96 53A6, and GROMOS96 54A7 produce results that fit best with experimental data, suggesting these may be better choices for working with other IDPs.

As stated previously, the crowded cellular environment can result in both excluded volume effect or protein-polymer interactions. Badasyan et al. describe a Hamiltonian that incorporates 1) competing interactions, where solute forms hydrogen bonds with a biopolymer, therefore competing for intra-polymer hydrogen bonding, and 2) non-competing interactions, where solute affects the solvent, which then affects the biopolymer. ${ }^{67}$ The Hamiltonian can account for a number of effects on a system with any biopolymer, and can more specifically help in understanding IDPs.

\section{Experimental approaches for the analysis of intrinsic disorder}

\section{NMR}

As previously discussed, traditional computational methods of determining protein folding interactions generally do not consider protein intrinsic disorder. Xia and Wei introduced molecular nonlinear dynamics (MND) as a novel method for representing protein folding and aggregation. ${ }^{68}$ The authors also introduce the term intrinsically low dimensional manifolds (ILDMs) as a characteristic of folded proteins, and show that this is useful for generating unfolding pathways and protein flexibility analysis.

Traditional (1)H-(15)N HSQC spectroscopy is not ideal for IDPs, as fast solvent exchange causes a decrease in spectral quality, and this effect is biased toward IDPs. Yuwen and Skrynnikov introduced cross-polarization assisted heteronuclear in-phase 
single-quantum correlation (CP-HISQC) as an alternative experimental method to circumvent this bias. ${ }^{69}$ CP-HISQC takes advantage of both proton-decoupled HSQC and cross-polarization ${ }^{1} \mathrm{H}$-to- ${ }^{15} \mathrm{~N}$ transfer to compensate for the loss of spectral quality caused by fast solvent exchange.

In order for time-resolved experiments to produce useful data, the length of data collection must agree with the time of the phenomena to be observed, which is generally problematic. Mayzel et al. described a method of using non-uniform sampling and fast-pulsing BEST-TROSY, combined with co-processing using multidimensional decomposition, to resolve this problem. ${ }^{70}$ The authors verify the technique by observing tyrosine phosphorylation of a disordered region of CD79b.

The proton dimension of NMR spectra generally low quality for IDPs, where chemical shifts are close to simply random coil values. Meyer and Zangger demonstrated the use of homonuclear broadband proton decoupling of multidimensional NMR, which had previously been proposed as a method of enhancing resolution of small molecules. ${ }^{71}$ The authors were able to incorporate this method by using a weak field pulsed gradient combined with frequency-selective pulses, and validated their method using 2 IDPs, $\alpha$-synuclein and SERF1a.

IDPs are often enriched in proline, a residue which can be problematic for NMR characterization due to its unusual side chain-peptide backbone interaction, which results in a lack of an amide proton. Hellman et al. proposed two new pulse sequences which were able to improve backbone assignments greater than $22 \%$ in the proline-rich $\mathrm{C}$-terminus of adenosine receptor $2 \mathrm{~A} .{ }^{72}$

Sahu et al. took a different approach to resolving the issues involved in analyzing IDPs in the proton spectrum. The authors used carbon-detected spectra of the Pdx1 C-terminal domain to generate several pulse protocols that do not depend on $4 \mathrm{D}$ or greater experiments or the previously mentioned non-uniform sampling. ${ }^{73}$

Proteins at membrane/water interfaces, such as phenylalanine-glycine (FG) repeat peptides of the nuclear pore complex, encounter a spectrum of solvent conditions including changes in dielectric constants and viscosity. Heisel and Krishnan used NMR combined with a solvent exchange scheme to examine the structural transitions of FG-peptides from DMSO to water, providing a basis for future co-solvent NMR experiments. $^{74}$

Although recombinant monomeric $\alpha$-synuclein is generally regarded to be an IDP, the native state of $\alpha$-synuclein in cells has been a matter of long-standing debate. Selkoe et al. reviewed their evidence that in cells, $\alpha$-synuclein exists as a helically folded tetramer, which dissociate upon non-crowding conditions. ${ }^{75}$ The authors also review the various methods and reagents used to reach their hypothesis.

Kadeřávek et al. examined (15)N reduced spectral density mapping protocols as they relate to IDPs. ${ }^{76}$ By incorporating cross-correlated relaxation rates, the authors were able to describe optimized methods of NMR analysis of IDPs. Using a model IDP, delta subunit of bacterial RNA polymerase, the authors were able to validate their approach as an unbiased method of analyzing IDP motions.

\section{Single-molecule spectroscopy}

Brucale et al. reviewed the study of IDPs using singlemolecule spectroscopy. ${ }^{77}$ This comprehensive literature analysis encompasses approximately a decade of research and approximately 200 articles. Among other topics, the authors cover introductory concepts, instrumentation, dyes, methods, and relevance to the field of IDPs. Finally, the authors speculate on the future directions of the field, in the context of how future developments can help answer pervading questions about IDPs.

Another review of single molecule fluorescence as it pertains to IDPs is provided by Banerjee and Deniz. ${ }^{78}$ This review takes a more focused approach, discussing single molecule studies as it relates to protein folding, free energy landscapes, and dynamics of IDPs.

\section{Recombinant production of IDPs}

Recombinant protein production in E. coli is easily the most widely used method of producing recombinant protein because of its relative ease and cost-efficiency; however, production of aggregation-prone proteins that are generally IDPs are challenging. A review by Lebendiker and Danieli addresses methods of reducing and preventing aggregate formation at every purification step from vector construction all the way to storage of purified protein. ${ }^{79}$

Cellular nucleic acid binding protein (CNBP) is involved in enhancing $c-m y c$ expression and binding to ssDNA and RNA. However, purification of tag-free 
protein has historically been challenging. Challier et al. described a method of high production of tag-free recombinant $\mathrm{CNBP}$, and characterized some of the physicochemical properties of this protein. ${ }^{80}$ The authors found that this protein is highly flexible with multiple structural zincs, and it gains structure upon binding to nucleic acid.

As IDPs lack stable structure, biologically relevant protein can be obtained from inclusion bodies when recombinantly produced in E.coli; therefore, this avenue may be preferable to prevent protease cleavage and degradation. Hwang et al. reviewed protein production strategies involving fusions of IDPs, as well as short peptides and proteins that are easily refolded, to a tag that is targeted toward inclusion bodies. ${ }^{81}$ The authors describe the tags necessary, the purification conditions, and cleavage methods to remove the fusion tag.

\section{Miscellaneous}

Divalent cations can significantly affect the compaction of IDPs as well as globular proteins. A minireview by Lee examined nanopore studies of numerous peptides and proteins which interact with divalent cations. ${ }^{17}$ Generally, metal ion binding results in compaction, and the effects of this on several IDPs such as prion, myelin basic protein, and a 30aa $\mathrm{Zn}$-finger peptide are described.

\section{Proteomics}

Xue and Uversky ${ }^{82}$ looked at the incidence of intrinsic disorder in 840 human antiviral proteins and highlighted the significant role that intrinsic disorder plays in the molecular arms race between viruses and host defense. Additionally, Xue et al. discussed the quantity and nature of intrinsic disorder in the interactome of the human papillomavirus. ${ }^{83}$

\section{Looking at IDPs/IDPRs in diseases}

\section{IDPs in neurodegenerative diseases}

The IDP $\alpha$-synuclein interacts with cellular membranes and is $\mathrm{N}$-terminally acetylated. However, a majority of lipid interaction studies use non-acetylated protein. A study by Dikiy and Eliezer examined the effects of acetylation on $\alpha$-synuclein interactions with lipid micelles. ${ }^{84}$ Using $\beta$-octylglucoside and SDS, a nonionic and anionic detergent respectively, the authors found that acetylation generally increases affinity of $\alpha$-synuclein for membranes, and that interaction with membranes is dependent on the charge composition of the lipid micelle in addition to the acetylation state.

Using single-tryptophan mutants of $\alpha$-synuclein to quantify insertion of the protein into lipid membranes, Shvadchak and Subramaniam found a 4 amino acid linker which is unnecessary for membrane interactions, but is necessary for efficient fibrillation. ${ }^{85}$ This flexible linker is between 2 of the 7 helical repeats in the protein, and is speculated to be a potential cause of clinical synucleinopathies.

$\alpha$-synuclein has been thoroughly examined in both monomeric and oligomeric forms. Lorenzen et al. examined various $\mathrm{N}$-terminal deletions of $\alpha$-synuclein, and found that the first 11 residues of both monomeric and oligomeric forms are important for folding into, binding, and permeabilization for phospholipid bilayers. ${ }^{86}$ However, the oligomeric form more efficiently inserts into bilayers, presumably because of exposed hydrophobic surfaces and electrostatic effects of the N-termini.

To better understand membrane insertion of $\alpha$-synuclein, Gurnev et al. examined this in the context of a lipid bilayer-reconstituted channel protein, $\alpha$-hemolysin. ${ }^{87}$ The authors found that blockage of the channel by $\alpha$-synuclein was dependent on the side of the channel that the $\alpha$-synuclein was added on, as well as the sidedness of the applied potential. Overall, the authors found that the system of a reconstituted pore protein could be used to capture different information about $\alpha$-synuclein, including translocation, membrane interactions, and protein state.

Numerous studies have been published characterizing the properties of recombinant $\alpha$-synuclein. A review by Narkiewicz et al. summarize the biophysical properties of recombinant $\alpha$-synuclein aggregation, the techniques used to gain this information, and the factors that mediate this process. ${ }^{88}$ Additionally, the authors describe the effects of clinically relevant mutations on these properties. Finally, they describe potential physiological effects of $\alpha$-synuclein aggregation on the cellular environment.

The role of the $\mathrm{A} \beta \mathrm{N}$-terminus on its aggregation is a topic of debate in the field. A study by Nguyen et al. examined an $\mathrm{A} 2 \mathrm{~V}$ variant using molecular dynamics simulations, and found that this mutation decreased overall level of intrinsic disorder and increased $\beta$-hairpin content, potentially providing an overall mechanism for the pathogenicity of this variant. ${ }^{89}$ 
Tau is an IDP that stabilizes microtubules, and forms tangles in the brain that are associated with neurotoxicity. Its cognate chaperone, Hsp90, can both stabilize and degrade tau by facilitating interactions of tau with microtubules and working with E3 ligases, respectively. A study by Karagöz et al. used a combination of NMR and SAXS to obtain a structural model of Hsp90 with tau, and found that the interaction interface is broad and contains a number of low affinity contacts, including dispersed hydrophobic residues characteristic of late folding intermediates. ${ }^{90}$ This study provides a mechanism of action as to how Hsp90 can select for IDPs as well as late folding intermediates.

TDP-43 aggregation is associated with several neuropathologies. A study by Udan-Johns et al. examined the physiological steps leading to TDP-43 aggregation, and found that the mechanism of action is comparable to yeast prion. ${ }^{91}$ Heat shock-induced reversible aggregation is mediated by the $\mathrm{C}$-terminus as well as available chaperones. Additionally, aggregation results in decreased interaction with hnRNP1, resulting in altered RNA-binding partners and thus function of TDP-43.

\section{Viral IDPs}

Human papilloma virus (HPV) infection is commonly known to induce several types of cancer, and the E7 oncoprotein targets the retinoblastoma $(\mathrm{Rb})$ tumor suppressor, among other targets. Chemes et al. examined the role of oxidative stress on E7, as this protein has a high percentage of cysteines. ${ }^{92}$ The authors used multiple biochemical and biophysical techniques, and found that oxidative stress causes formation of a disulfide bridge, resulting in the first observed interaction between the disordered $\mathrm{N}$-terminus and the globular C-terminus.

TRIM5 $\alpha$ is a restriction factor that blocks retroviral replication of HIV-1 through its C-terminal SPRY domain. A long disordered v1 loop in this SPRY domain was examined by Kovalskyy and Ivanon through all-atom molecular dynamics simulations. ${ }^{93}$ The authors found that the v1 loop samples multiple conformations which allows for several binding poses with the HIV-1 capsid, providing a potential mechanism where the disordered v1 loop facilitates TRIM5 $\alpha$ 's ability to effect HIV-1 despite the high frequency of evasion mutations in the capsid.

The SARS coronavirus nucleocapsid $(\mathrm{N})$ protein functions by packaging the genetic material into the viral capsid, and is therefore important for self-assembly. This protein has multiple disordered regions, and a review by Chang et al. describes the structural and allosteric characteristics of the $\mathrm{N}$ protein as it relates to intrinsic disorder. ${ }^{94}$ The authors propose that intrinsic disorder is essential to RNP packaging and viral self-assembly.

\section{Infectious disease}

Valizadeh et al. collected several Plasmodium vivax isolates to characterize the SNPs of Region II of Duffy binding protein (PvDBP-II), a potential target of a malaria vaccine. ${ }^{95}$ The authors found that a region of the protein which is intrinsically disordered was highly polymorphic, and positive selective pressure acts on this region. This study provides a potential structural explanation for the genetic diversity of this protein.

\section{IDPs/IDPRs as drugs or drug targets}

Campadre et al. demonstrate that flexibility in drug design can be an advantage with the design of vitamin E analogs, called tocoflexols. ${ }^{96}$ They showed using MD simulations that compounds with a flexible tail have better binding affinity to the $\alpha$-tocopherol transfer protein (ATTP) and therefore have longer plasma half-lives. Tocoflexols have the potential to be safe radioprotection/radiomitigation agents for use in radiological emergencies.

IDPs are increasingly being recognized as valid drug targets. Chu et al. ${ }^{97}$ discuss the targeting of an intrinsically disordered extracellular domain of the membrane bound $\operatorname{IgE}$ (mIgE), an antibody that is involved in hypersensitivity reactions in allergic diseases. They show that 2 different antibodies target different conformations of this IDR and downnregulate IgE preduction.

Disordered regions have been implicated in multiple disease processes. Charpentier et al. ${ }^{98}$ examine the highly flexible microtentacles (McTN) that protrude from the cell membrane. They show that cancer stemlike cells (CSC) have a large number of McTNs and that natural compound curcumin reduced McTN in breast CSC. While the exact mechanism has not yet been elucidated, they propose that curcumin may bind directly to tubulin or may interfere with microtubule dynamics. 


\section{Disclosure of potential conflicts of interest}

No potential conflicts of interest were disclosed.

\section{References}

[1] Uversky VN. Digested disorder: Quarterly intrinsic disorder digest (January/February/ March, 2013). Intrinsically Dis Proteins 2013; 1:e25496; http://dx.doi.org/10.4161/ idp. 25496

[2] DeForte S, Reddy KD, Uversky VN. Digested disorder, issue \#2: Quarterly intrinsic disorder digest (April/May/ June, 2013). Intrinsically Dis Proteins 2013; 1:e27454; http://dx.doi.org/10.4161/idp.27454

[3] Reddy KD, DeForte S, Uversky VN. Digested disorder, issue \#3: Quarterly intrinsic disorder digest (July/August/ September, 2013). Intrinsically Dis Proteins 2013; 2: e27833; http://dx.doi.org/10.4161/idp.27833

[4] DeForte S, Reddy KD, Uversky VN. Digested disorder, issue \#4: Quarterly intrinsic disorder digest (October-November-December, 2013). Intrinsically Dis Proteins 2015; 3 1-10; http://dx.doi.org/10.4161/ 21690707.2014.984569

[5] Sickmeier M, Hamilton JA, LeGall T, Vacic V, Cortese MS, Tantos A, Szabo B, Tompa P, Chen J, Uversky VN, Obradovic Z, Dunker AK. DisProt: the Database of Disordered Proteins. Nucleic Acids Res 2007; 35:D786-93; PMID:17145717; http://dx.doi.org/10.1093/nar/gkl893

[6] Fukuchi S, Amemiya T, Sakamoto S, Nobe Y, Hosoda K, Kado Y, Murakami SD, Koike R, Hiroaki H, Ota M. IDEAL in 2014 illustrates interaction networks composed of intrinsically disordered proteins and their binding partners. Nucleic Acids Res 2014; 42:D320-5; PMID:24178034; http://dx.doi.org/10.1093/nar/gkt1010

[7] Oates ME, Romero P, Ishida T, Ghalwash M, Mizianty MJ, Xue B, Dosztányi Z, Uversky VN, Obradovic Z, Kurgan $\mathrm{L}$, et al. $\mathrm{D}^{2} \mathrm{P}^{2}$ : database of disordered protein predictions. Nucleic Acids Res 2013; 41:D508-16; PMID:23203878; http://dx.doi.org/10.1093/nar/gks1226

[8] Di Domenico T, Walsh I, Tosatto SC. Analysis and consensus of currently available intrinsic protein disorder annotation sources in the MobiDB database. BMC Bioinformatics 2013; 14 Suppl 7:S3; PMID:23815411; http:// dx.doi.org/10.1186/1471-2105-14-S7-S3

[9] Varadi M, Kosol S, Lebrun P, Valentini E, Blackledge M, Dunker AK, Felli IC, Forman-Kay JD, Kriwacki RW, Pierattelli R, et al. pE-DB: a database of structural ensembles of intrinsically disordered and of unfolded proteins. Nucleic Acids Res 2014; 42:D326-35; PMID:24174539; http://dx.doi.org/10.1093/nar/gkt960

[10] Tompa P, Varadi M. Predicting the predictive power of IDP ensembles. Structure 2014; 22:177-8; PMID:24507778; http://dx.doi.org/10.1016/j.str.2014.01.003

[11] Schwalbe M, Ozenne V, Bibow S, Jaremko M, Jaremko L, Gajda M, Jensen MR, Biernat J, Becker S, Mandelkow E, et al. Predictive atomic resolution descriptions of intrinsically disordered hTau40 and alpha-synuclein in solution from NMR and small angle scattering. Structure 2014; 22:238-49; PMID:24361273; http://dx.doi.org/10.1016/j. str.2013.10.020

[12] Heinrich J, Krone M, O’Donoghue SI, Weiskopf D. Visualising intrinsic disorder and conformational variation in protein ensembles. Faraday Discuss 2014; 169:179-93; PMID:25340810; http://dx.doi.org/10.1039/C3FD00138E

[13] De Biasio A, Ibanez de Opakua A, Cordeiro TN, Villate M, Merino N, Sibille N, Lelli M, Diercks T, Bernado P, Blanco FJ. p15PAF is an intrinsically disordered protein with nonrandom structural preferences at sites of interaction with other proteins. Biophysical journal 2014; 106:865-74; PMID:24559989; http://dx.doi.org/10.1016/j. bpj.2013.12.046

[14] Sheikh MO, Schafer CM, Powell JT, Rodgers KK, Mooers $\mathrm{BH}$, West CM. Glycosylation of Skp1 affects its conformation and promotes binding to a model f-box protein. Biochemistry 2014; 53:1657-69; PMID:24506136; http:// dx.doi.org/10.1021/bi401707y

[15] Keller JP, Homma K, Duan C, Zheng J, Cheatham MA, Dallos P. Functional regulation of the SLC26-family protein prestin by calcium/calmodulin. J Neurosci 2014; 34:1325-32; PMID:24453323; http://dx.doi.org/10.1523/ JNEUROSCI.4020-13.2014

[16] Iesmantavicius V, Dogan J, Jemth P, Teilum K, Kjaergaard M. Helical propensity in an intrinsically disordered protein accelerates ligand binding. Angewandte Chemie 2014; 53:1548-51; PMID:24449148; http://dx.doi.org/ 10.1002/anie.201307712

[17] Lee JS. Nanopore analysis of the effect of metal ions on the folding of peptides and proteins. Protein and peptide letters 2014; 21:247-55; PMID:24370255; http://dx.doi. org/10.2174/09298665113209990075

[18] Golebiewska U, Zurawsky C, Scarlata S. Defining the oligomerization state of gamma-synuclein in solution and in cells. Biochemistry 2014; 53:293-9; PMID:24367999; http://dx.doi.org/10.1021/bi401479a

[19] Brister MA, Pandey AK, Bielska AA, Zondlo NJ. OGlcNAcylation and phosphorylation have opposing structural effects in tau: phosphothreonine induces particular conformational order. J Am Chem Soc 2014; 136:380316; PMID:24559475; http://dx.doi.org/10.1021/ ja407156m

[20] Grishin AM, Cherney M, Anderson DH, Phanse S, Babu $\mathrm{M}$, Cygler M. NleH defines a new family of bacterial effector kinases. Structure 2014; 22:250-9; PMID:24373767; http://dx.doi.org/10.1016/j.str.2013.11.006

[21] Deshmukh L, Ghirlando R, Clore GM. Investigation of the structure and dynamics of the capsid-spacer peptide 1-nucleocapsid fragment of the HIV-1 gag polyprotein by solution NMR spectroscopy. Angewandte Chemie 2014; 53:1025-8; PMID:24338988; http://dx.doi.org/ 10.1002/anie.201309127

[22] Martinez-Zapien D, Delsuc MA, Trave G, Lutzing R, Rochette-Egly C, Kieffer B. Production and characterization of a retinoic acid receptor RARgamma construction encompassing the DNA binding domain and the 
disordered N-terminal proline rich domain. Protein Exp Purificat 2014; 95:113-20; PMID:24333369; http://dx.doi. org/10.1016/j.pep.2013.12.001

[23] Nourse A, Mittag T. The cytoplasmic domain of the Tcell receptor zeta subunit does not form disordered dimers. J Mol Biol 2014; 426:62-70; PMID:24120941; http://dx.doi.org/10.1016/j.jmb.2013.09.036

[24] Hill SA, Kwa LG, Shammas SL, Lee JC, Clarke J. Mechanism of assembly of the non-covalent spectrin tetramerization domain from intrinsically disordered partners. J Mol Biol 2014; 426:21-35; PMID:24055379; http://dx.doi. org/10.1016/j.jmb.2013.08.027

[25] Butz M, Kast P, Hilvert D. Affinity maturation of a computationally designed binding protein affords a functional but disordered polypeptide. J Struct Biol 2014; 185:168-77; PMID:23537847; http://dx.doi.org/10.1016/j. jsb.2013.03.008

[26] Hashi Y, Kawai G, Kotani S. Microtubule-associated protein (MAP) 4 interacts with microtubules in an intrinsically disordered manner. Biosci Biotechnol Biochem 2014; 78:1864-70; PMID:25052097; http://dx.doi.org/ 10.1080/09168451.2014.940836

[27] Breydo L, Reddy KD, Piai A, Felli IC, Pierattelli R, Uversky VN. The crowd you're in with: effects of different types of crowding agents on protein aggregation. Biochimica et biophysica acta 2014; 1844:346-57; PMID:24252314; http://dx.doi.org/ 10.1016/j.bbapap.2013.11.004

[28] Kutyshenko VP, Prokhorov DA, Molochkov NV, Sharapov MG, Kolesnikov I, Uversky VN. Dancing retro: solution structure and micelle interactions of the retro-SH3domain, retro-SHH-'Bergerac'. J Biomol Struct Dynam 2014; 32:257-72; PMID:23527530; http://dx.doi.org/ $10.1080 / 07391102.2012 .762724$

[29] Hendus-Altenburger R, Kragelund BB, Pedersen SF. Structural dynamics and regulation of the mammalian SLC9A family of $\mathrm{Na}(+) / \mathrm{H}(+)$ exchangers. Curr Topics Membranes 2014; 73:69-148; PMID:24745981; http://dx. doi.org/10.1016/B978-0-12-800223-0.00002-5

[30] Oldfield CJ, Dunker AK. Intrinsically disordered proteins and intrinsically disordered protein regions. Annu Rev Biochem 2014; 83:553-84; PMID:24606139; http://dx.doi. org/10.1146/annurev-biochem-072711-164947

[31] Hisaoka M, Nagata K, Okuwaki M. Intrinsically disordered regions of nucleophosmin/B23 regulate its RNA binding activity through their inter- and intra-molecular association. Nucleic Acids Res 2014; 42:1180-95; PMID:24106084; http://dx.doi.org/10.1093/nar/gkt897

[32] Quan S, Wang L, Petrotchenko EV, Makepeace KA, Horowitz S, Yang J, Zhang Y, Borchers CH, Bardwell JC. Super Spy variants implicate flexibility in chaperone action. Elife 2014; 3:e01584; PMID:24497545; http://dx. doi.org/10.7554/eLife.01584

[33] Bencivenga D, Tramontano A, Borgia A, Negri A, Caldarelli I, Oliva A, Perrotta S, Della Ragione F, Borriello A. p27Kip1 serine 10 phosphorylation determines its metabolism and interaction with cyclin-dependent kinases. Cell Cycle 2014; 13:3768-82; PMID:25483085; http://dx.doi.org/10.4161/15384101.2014.965999

[34] Khurana J, Manisha, Singh R, Kaur J. Intrinsically unstructured carboxy terminus of Bacillus lipase is essential for its function. Protein Pept Lett 2014; 21:1265-72; PMID:25001212

[35] Choy MS, Hieke M, Kumar GS, Lewis GR, GonzalezDeWhitt KR, Kessler RP, Stein BJ, Hessenberger M, Nairn AC, Peti W, et al. Understanding the antagonism of retinoblastoma protein dephosphorylation by PNUTS provides insights into the PP1 regulatory code. Proc Natl Acad Sci U S A 2014; 111:4097-102; PMID:24591642; http://dx.doi.org/10.1073/pnas.1317395111

[36] Hill AT, Ying S, Plaxton WC. Phosphorylation of bacterial-type phosphoenolpyruvate carboxylase by a $\mathrm{Ca} 2+-$ dependent protein kinase suggests a link between $\mathrm{Ca} 2+$ signalling and anaplerotic pathway control in developing castor oil seeds. Biochem J 2014; 458:109-18; PMID:24266766; http://dx.doi.org/10.1042/BJ20131191

[37] Rajagopalan K, Qiu R, Mooney SM, Rao S, Shiraishi T, Sacho E, Huang H, Shapiro E, Weninger KR, Kulkarni P. The Stress-response protein prostate-associated gene 4 , interacts with c-Jun and potentiates its transactivation. Biochim Biophys Acta 2014; 1842:154-63; PMID:24263171; http://dx.doi.org/ 10.1016/j.bbadis.2013.11.014

[38] Mooney SM, Qiu R, Kim JJ, Sacho EJ, Rajagopalan K, Johng D, Shiraishi T, Kulkarni P, Weninger KR. Cancer/ testis antigen PAGE4, a regulator of c-Jun transactivation, is phosphorylated by homeodomain-interacting protein kinase 1, a component of the stress-response pathway. Biochemistry 2014; 53:1670-9; PMID:24559171; http://dx.doi.org/10.1021/bi500013w

[39] Lee DW, Banquy X, Kristiansen K, Kaufman Y, Boggs JM, Israelachvili JN. Lipid domains control myelin basic protein adsorption and membrane interactions between model myelin lipid bilayers. Proc Natl Acad Sci U S A 2014; 111:E768-75; PMID:24516125; http://dx.doi.org/ 10.1073/pnas.1401165111

[40] Ramos I, Fernandez-Rivero N, Arranz R, Aloria K, Finn R, Arizmendi JM, Ausio J, Valpuesta JM, Muga A, Prado A. The intrinsically disordered distal face of nucleoplasmin recognizes distinct oligomerization states of histones. Nucleic Acids Res 2014; 42:1311-25; PMID:24121686; http://dx.doi.org/10.1093/nar/gkt899

[41] Albar AH, Almehdar HA, Uversky VN, Redwan EM. Structural heterogeneity and multifunctionality of lactoferrin. Curr Protein Pept Sci 2014; 15:77897; PMID:25245670; http://dx.doi.org/10.2174/ 1389203715666140919124530

[42] Simons SS, Jr., Edwards DP, Kumar R. Minireview: dynamic structures of nuclear hormone receptors: new promises and challenges. Mol Endocrinol 2014; 28:17382; PMID:24284822; http://dx.doi.org/10.1210/me.20131334

[43] Fiserova J, Spink M, Richards SA, Saunter C, Goldberg MW. Entry into the nuclear pore complex is controlled 
by a cytoplasmic exclusion zone containing dynamic GLFG-repeat nucleoporin domains. J Cell Sci 2014; 127:124-36; PMID:24144701; http://dx.doi.org/10.1242/ jcs. 133272

[44] Calidas D, Lyon H, Culver GM. The N-terminal extension of S12 influences small ribosomal subunit assembly in Escherichia coli. Rna 2014; 20:321-30; PMID:24442609; http://dx.doi.org/10.1261/rna.042432.113

[45] Asano N, Atsuumi H, Nakamura A, Tanaka Y, Tanaka I, Yao M. Direct interaction between EFL1 and SBDS is mediated by an intrinsically disordered insertion domain. Biochem Biophys Res Commun 2014; 443:1251-6; PMID:24406167; http://dx.doi.org/ 10.1016/j.bbrc.2013.12.143

[46] Erales J, Coffino P. Ubiquitin-independent proteasomal degradation. Biochim Biophys Acta 2014; 1843:216-21; PMID:23684952; http://dx.doi.org/ 10.1016/j.bbamcr.2013.05.008

[47] Tomko RJ, Jr., Hochstrasser M. The intrinsically disordered Sem1 protein functions as a molecular tether during proteasome lid biogenesis. Mol Cell 2014; 53:433-43; PMID:24412063; http://dx.doi.org/10.1016/ j.molcel.2013.12.009

[48] Striebel F, Imkamp F, Ozcelik D, Weber-Ban E. Pupylation as a signal for proteasomal degradation in bacteria. Biochim Biophys Acta 2014; 1843:10313; PMID:23557784; http://dx.doi.org/10.1016/j. bbamcr.2013.03.022

[49] Christian H, Hofele RV, Urlaub H, Ficner R. Insights into the activation of the helicase Prp43 by biochemical studies and structural mass spectrometry. Nucleic Acids Res 2014; 42:1162-79; PMID:24165877; http://dx.doi.org/ 10.1093/nar/gkt985

[50] Thakur JK, Yadav A, Yadav G. Molecular recognition by the KIX domain and its role in gene regulation. Nucleic Acids Res 2014; 42:2112-25; PMID:24253305; http://dx. doi.org/10.1093/nar/gkt1147

[51] Jiao Y, Walker M, Trinick J, Pernier J, Montaville P, Carlier MF. Mutagenetic and electron microscopy analysis of actin filament severing by Cordon-Bleu, a WH2 domain protein. Cytoskeleton (Hoboken) 2014; 71:170-83; PMID:24415668; http://dx.doi.org/10.1002/cm.21161

[52] Lyabin DN, Eliseeva IA, Ovchinnikov LP. YB-1 protein: functions and regulation. Wiley Interdiscip Rev RNA 2014; 5:95-110; PMID:24217978; http://dx.doi.org/ 10.1002/wrna.1200

[53] Jemth P, Mu X, Engstrom A, Dogan J. A frustrated binding interface for intrinsically disordered proteins. J Biol Chem 2014; 289:5528-33; PMID:24421312; http://dx.doi. org/10.1074/jbc.M113.537068

[54] Marsh JA, Teichmann SA. Parallel dynamics and evolution: Protein conformational fluctuations and assembly reflect evolutionary changes in sequence and structure. Bioessays 2014; 36:209-18; PMID:24272815; http://dx. doi.org/10.1002/bies.201300134

[55] Lee C, Kalmar L, Xue B, Tompa P, Daughdrill GW, Uversky VN, Han KH. Contribution of proline to the pre- structuring tendency of transient helical secondary structure elements in intrinsically disordered proteins. Biochim Biophys Acta 2014; 1840:993-1003; PMID:24211251; http://dx.doi.org/10.1016/j.bbagen.2013.10.042

[56] Lobanov MY, Galzitskaya OV. Occurrence of disordered patterns and homorepeats in eukaryotic and bacterial proteomes. Mol Biosyst 2012; 8:327-37; PMID:22009164; http://dx.doi.org/10.1039/C1MB05318C

[57] Lobanov MY, Sokolovskiy IV, Galzitskaya OV. HRaP: database of occurrence of HomoRepeats and patterns in proteomes. Nucleic Acids Res 2014; 42:D273-8; PMID:24150944; http://dx.doi.org/10.1093/nar/gkt927

[58] Finn RD, Bateman A, Clements J, Coggill P, Eberhardt RY, Eddy SR, Heger A, Hetherington K, Holm L, Mistry J, et al. Pfam: the protein families database. Nucleic Acids Res 2014; 42:D222-30; PMID:24288371; http://dx.doi. org/10.1093/nar/gkt1223

[59] Peng Z, Mizianty MJ, Kurgan L. Genome-scale prediction of proteins with long intrinsically disordered regions. Proteins 2014; 82:145-58; PMID:23798504; http://dx.doi. org/10.1002/prot.24348

[60] Fan X, Kurgan L. Accurate prediction of disorder in protein chains with a comprehensive and empirically designed consensus. J Biomol Struct Dyn 2014; 32:44864; PMID:23534882; http://dx.doi.org/10.1080/ 07391102.2013.775969

[61] Huang YJ, Acton TB, Montelione GT. DisMeta: a meta server for construct design and optimization. Methods Mol Biol 2014; 1091:3-16; PMID:24203321; http://dx.doi. org/10.1007/978-1-62703-691-7_1

[62] Monastyrskyy B, Kryshtafovych A, Moult J, Tramontano A, Fidelis K. Assessment of protein disorder region predictions in CASP10. Proteins 2014; 82 Suppl 2:127-37; PMID:23946100; http://dx.doi.org/10.1002/prot.24391

[63] Higo J, Umezawa K. Free-energy landscape of intrinsically disordered proteins investigated by all-atom multicanonical molecular dynamics. Adv Exp Med Biol 2014; 805:331-51; PMID:24446368; http://dx.doi.org/10.1007/ 978-3-319-02970-2_14

[64] Wang J, Shao Q, Xu Z, Liu Y, Yang Z, Cossins BP, Jiang $\mathrm{H}$, Chen K, Shi J, Zhu W. Exploring transition pathway and free-energy profile of large-scale protein conformational change by combining normal mode analysis and umbrella sampling molecular dynamics. J Phys Chem B 2014; 118:134-43; PMID:24350625; http://dx.doi.org/ $10.1021 / \mathrm{jp} 4105129$

[65] Rawat N, Biswas P. Hydrogen bond dynamics in intrinsically disordered proteins. J Phys Chem B 2014; 118:301825; PMID:24571104; http://dx.doi.org/10.1021/jp5013544

[66] Gerben SR, Lemkul JA, Brown AM, Bevan DR. Comparing atomistic molecular mechanics force fields for a difficult target: a case study on the Alzheimer's amyloid betapeptide. J Biomol Struct Dyn 2014; 32:181732; PMID:24028075; http://dx.doi.org/10.1080/ 07391102.2013 .838518

[67] Badasyan A, Tonoyan SA, Giacometti A, Podgornik R, Parsegian VA, Mamasakhlisov Y, Morozov VF. Unified 
description of solvent effects in the helix-coil transition. Phys Rev E, Statist Nonlin Soft Matter Phys 2014; 89:022723; PMID:25353524; http://dx.doi.org/10.1103/ PhysRevE.89.022723

[68] Xia K, Wei GW. Molecular nonlinear dynamics and protein thermal uncertainty quantification. Chaos 2014; 24:013103; PMID:24697365; http://dx.doi.org/10.1063/ 1.4861202

[69] Yuwen T, Skrynnikov NR. CP-HISQC: a better version of HSQC experiment for intrinsically disordered proteins under physiological conditions. J Biomol NMR 2014; 58:175-92; PMID:24496557; http://dx.doi.org/10.1007/ s10858-014-9815-5

[70] Mayzel M, Rosenlow J, Isaksson L, Orekhov VY. Timeresolved multidimensional NMR with non-uniform sampling. J Biomol NMR 2014; 58:129-39; PMID:24435565; http://dx.doi.org/10.1007/s10858-013-9811-1

[71] Meyer NH, Zangger K. Enhancing the resolution of multi-dimensional heteronuclear NMR spectra of intrinsically disordered proteins by homonuclear broadband decoupling. Chem Commun 2014; 50:1488-90; PMID:24366473; http://dx.doi.org/10.1039/C3CC48135B

[72] Hellman M, Piirainen H, Jaakola VP, Permi P. Bridge over troubled proline: assignment of intrinsically disordered proteins using (HCA)CON(CAN)H and (HCA)N (CA) $\mathrm{CO}(\mathrm{N}) \mathrm{H}$ experiments concomitantly with $\mathrm{HNCO}$ and i(HCA)CO(CA)NH. J Biomol NMR 2014; 58:49-60; PMID:24346685; http://dx.doi.org/10.1007/s10858-0139804-0

[73] Sahu D, Bastidas M, Showalter SA. Generating NMR chemical shift assignments of intrinsically disordered proteins using carbon-detected NMR methods. Anal Biochem 2014; 449:17-25; PMID:24333248; http://dx.doi. org/10.1016/j.ab.2013.12.005

[74] Heisel KA, Krishnan VV. NMR based solvent exchange experiments to understand the conformational preference of intrinsically disordered proteins using FG-nucleoporin peptide as a model. Biopolymers 2014; 102:69-77; PMID:24037535; http://dx.doi. org/10.1002/bip.22402

[75] Selkoe D, Dettmer U, Luth E, Kim N, Newman A, Bartels T. Defining the native state of alpha-synuclein. NeuroDegenerat Dis 2014; 13:114-7; PMID:24192542; http:// dx.doi.org/10.1159/000355516

[76] Kaderavek P, Zapletal V, Rabatinova A, Krasny L, Sklenar V, Zidek L. Spectral density mapping protocols for analysis of molecular motions in disordered proteins. J Biomol NMR 2014; 58:193-207; PMID:24515886; http:// dx.doi.org/10.1007/s10858-014-9816-4

[77] Brucale M, Schuler B, Samori B. Single-molecule studies of intrinsically disordered proteins. Chem Rev 2014; 114:3281-317; PMID:24432838; http://dx.doi.org/ $10.1021 / \mathrm{cr} 400297 \mathrm{~g}$

[78] Banerjee PR, Deniz AA. Shedding light on protein folding landscapes by single-molecule fluorescence. Chem Soc Rev 2014; 43:1172-88; PMID:24336839; http://dx.doi. org/10.1039/C3CS60311C
[79] Lebendiker M, Danieli T. Production of prone-to-aggregate proteins. FEBS Lett 2014; 588:23646; PMID:24211444; http://dx.doi.org/10.1016/j.febslet. 2013.10.044

[80] Challier E, Lisa MN, Nerli BB, Calcaterra NB, Armas P. Novel high-performance purification protocol of recombinant CNBP suitable for biochemical and biophysical characterization. Prot Exp Purificat 2014; 93:2331; PMID:24161561; http://dx.doi.org/10.1016/j.pep. 2013.10.006

[81] Hwang PM, Pan JS, Sykes BD. Targeted expression, purification, and cleavage of fusion proteins from inclusion bodies in Escherichia coli. FEBS Lett 2014; 588:24752; PMID:24076468; http://dx.doi.org/10.1016/j.febslet. 2013.09.028

[82] Xue B, Uversky VN. Intrinsic disorder in proteins involved in the innate antiviral immunity: another flexible side of a molecular arms race. J Mol Biol 2014; 426:1322-50; PMID:24184279; http://dx.doi.org/10.1016/ j.jmb.2013.10.030

[83] Xue B, Ganti K, Rabionet A, Banks L, Uversky VN. Disordered interactome of human papillomavirus. Curr Pharm Des 2014; 20:1274-92; PMID:23713779; http://dx. doi.org/10.2174/13816128113199990072

[84] Dikiy I, Eliezer D. N-terminal acetylation stabilizes Nterminal helicity in lipid- and micelle-bound alpha-synuclein and increases its affinity for physiological membranes. J Biol Chem 2014; 289:3652-65; PMID:24338013; http://dx.doi.org/10.1074/jbc.M113.512459

[85] Shvadchak VV, Subramaniam V. A four-amino acid linker between repeats in the alpha-synuclein sequence is important for fibril formation. Biochemistry 2014; 53:279-81; PMID:24397337; http://dx.doi.org/10.1021/ bi401427t

[86] Lorenzen N, Lemminger L, Pedersen JN, Nielsen SB, Otzen DE. The N-terminus of alpha-synuclein is essential for both monomeric and oligomeric interactions with membranes. FEBS Lett 2014; 588:497502; PMID:24374342; http://dx.doi.org/10.1016/j.febslet. 2013.12.015

[87] Gurnev PA, Yap TL, Pfefferkorn CM, Rostovtseva TK, Berezhkovskii AM, Lee JC, Parsegian VA, Bezrukov SM. Alpha-synuclein lipid-dependent membrane binding and translocation through the alpha-hemolysin channel. Biophy J 2014; 106:556-65; PMID:24507596; http://dx.doi. org/10.1016/j.bpj.2013.12.028

[88] Narkiewicz J, Giachin G, Legname G. In vitro aggregation assays for the characterization of alpha-synuclein prionlike properties. Prion 2014; 8:19-32; PMID:24552879; http://dx.doi.org/10.4161/pri.28125

[89] Nguyen PH, Tarus B, Derreumaux P. Familial Alzheimer A2 $\mathrm{V}$ mutation reduces the intrinsic disorder and completely changes the free energy landscape of the Abeta1-28 monomer. J Phys Chem B 2014; 118:501-10; PMID:24372615; http://dx.doi.org/10.1021/jp4115404

[90] Karagoz GE, Duarte AM, Akoury E, Ippel H, Biernat J, Moran Luengo T, Radli M, Didenko T, Nordhues BA, 
Veprintsev DB, et al. Hsp90-Tau complex reveals molecular basis for specificity in chaperone action. Cell 2014; 156:963-74; PMID:24581495; http://dx.doi.org/10.1016/j. cell.2014.01.037

[91] Udan-Johns M, Bengoechea R, Bell S, Shao J, Diamond MI, True HL, Weihl CC, Baloh RH. Prion-like nuclear aggregation of TDP-43 during heat shock is regulated by HSP40/70 chaperones. Hum Mol Gen 2014; 23:157-70; PMID:23962724; http://dx.doi.org/10.1093/hmg/ddt408

[92] Chemes LB, Camporeale G, Sanchez IE, de Prat-Gay G, Alonso LG. Cysteine-rich positions outside the structural zinc motif of human papillomavirus E7 provide conformational modulation and suggest functional redox roles. Biochemistry 2014; 53:1680-96; PMID:24559112; http:// dx.doi.org/10.1021/bi401562e

[93] Kovalskyy DB, Ivanov DN. Recognition of the HIV capsid by the TRIM5alpha restriction factor is mediated by a subset of pre-existing conformations of the TRIM5alpha SPRY domain. Biochemistry 2014; 53:1466-76; PMID:24506064; http://dx.doi.org/10.1021/bi4014962

[94] Chang CK, Hou MH, Chang CF, Hsiao CD, Huang TH. The SARS coronavirus nucleocapsid protein-forms and functions. Antivi Res 2014; 103:39-50; PMID:24418573; http://dx.doi.org/10.1016/j.antiviral.2013.12.009
[95] Valizadeh V, Zakeri S, Mehrizi AA, Djadid ND. Population genetics and natural selection in the gene encoding the Duffy binding protein II in Iranian Plasmodium vivax wild isolates. Infect Gen Evol 2014; 21:42435; PMID:24384095; http://dx.doi.org/10.1016/j.meegid. 2013.12.012

[96] Compadre CM, Singh A, Thakkar S, Zheng G, Breen PJ, Ghosh S, Kiaei M, Boerma M, Varughese KI, Hauer-Jensen M. Molecular dynamics guided design of tocoflexol: a new radioprotectant tocotrienol with enhanced bioavailability. Drug Dev Res 2014; 75:10-22; PMID:24648045; http://dx.doi.org/10.1002/ddr.21162

[97] Chu HM, Wright J, Chan YH, Lin CJ, Chang TW, Lim C. Two potential therapeutic antibodies bind to a peptide segment of membrane-bound IgE in different conformations. Nat Commun 2014; 5:3139; PMID:24457896

[98] Charpentier MS, Whipple RA, Vitolo MI, Boggs AE, Slovic J, Thompson KN, Bhandary L, Martin SS. Curcumin targets breast cancer stem-like cells with microtentacles that persist in mammospheres and promote reattachment. Cancer Res 2014; 74:1250-60; PMID:24371229; http://dx.doi.org/10.1158/0008-5472. CAN-13-1778 